\title{
Predicting the Need for an Implantable Cardioverter Defibrillator Using Cardiac Metaiodobenzylguanidine Activity Together with Plasma Natriuretic Peptide Concentration or Left Ventricular Function
}

\author{
Daigo Nagahara, Tomoaki Nakata, Akiyoshi Hashimoto, Takeru Wakabayashi, Michifumi Kyuma, Ryosuke Noda, \\ Shinya Shimoshige, Kikuya Uno, Kazufumi Tsuchihashi, and Kazuaki Shimamoto \\ Second Department of Internal Medicine (Cardiology), Sapporo Medical University School of Medicine, Sapporo, Japan
}

Despite widespread use of implantable cardioverter defibrillators (ICDs), their cost and the fact that only a certain group of patients fully benefits from the devices require appropriate risk stratification of patients. This study investigated whether altered cardiac autonomic function is associated with the occurrence of ICD discharge or lethal cardiac events. Methods: Fifty-four ICD-treated patients were prospectively followed after assessment of cardiac metaiodobenzylguanidine (MIBG) activity, quantified as the heartto-mediastinum ratio (HMR), plasma concentration of brain natriuretic peptide (BNP), and left ventricular ejection fraction (LVEF). Patients were divided into 2 groups based on the presence (group A, $n=21$ ) or absence (group B, $n=33$ ) of appropriate ICD discharge during a 15-mo period. Results: Group A had a significantly lower level of MIBG activity and a higher plasma BNP level than did group B. Univariate analysis revealed BNP level, any medication, and late HMR to be significant predictors, and multivariate analysis showed late HMR to be an independent predictor. An HMR of less than 1.95 with a plasma BNP level of more than $187 \mathrm{pg} / \mathrm{mL}$ or an LVEF of less than $50 \%$ had significantly increased power to predict ICD shock: positive predictive values, 82\% (HMR + BNP) and 58\% (HMR + LVEF); negative predictive values, $73 \%$ $(\mathrm{HMR}+\mathrm{BNP})$ and $77 \%$ (HMR + LVEF); sensitivities, 45\% (HMR + BNP) and 67\% (HMR + LVEF); and specificities, 94\% (HMR + BNP) and 70\% (HMR + LVEF). Conclusion: When combined with plasma BNP concentration or cardiac function, cardiac MIBG activity is closely related to lethal cardiac events and can be used to identify patients who would benefit most from an ICD.

Key Words: implantable cardioverter defibrillator; metaiodobenzylguanidine imaging; brain natriuretic peptide; sudden cardiac death; lethal arrhythmias

J Nucl Med 2008; 49:225-233

DOI: 10.2967/jnumed.107.042564

\footnotetext{
Received Apr. 6, 2007; revision accepted Sep. 10, 2007.

For correspondence or reprints contact: Daigo Nagahara, MD, Second Department of Internal Medicine (Cardiology), Sapporo Medical University School of Medicine, S-1, W-16, Chuo-ku, Sapporo 060-0061, Japan.

E-mail: dnagahar@sapmed.ac.jp

COPYRIGHT @ 2008 by the Society of Nuclear Medicine, Inc.
}

$\mathbf{I}_{n}$ mplantable cardioverter defibrillators (ICDs), which can abort potentially fatal ventricular arrhythmias, are widely used and improve the survival of patients with lethal arrhythmias and left ventricular dysfunction. ICD use is associated with a risk reduction rate of nearly $30 \%(1,2)$. The MADIT-II study (2), however, showed that the actual reduction of fatal events was by 5.6 percentage points (from $19.8 \%$ to $14.2 \%$ ). Given the high costs of ICD implantation and long-term management, the cost-effectiveness of this device could be improved and the number of adverse effects reduced if patients with a high likelihood of sudden cardiac death who could benefit most from this device were selected.

Several studies have shown that altered systemic or cardiac autonomic nervous function is related to the triggering or exacerbation of heart failure and the ventricular arrhythmias responsible for lethal cardiac events. Autonomic nervous function has both favorable (compensatory) and unfavorable effects on cardiovascular diseases and outcomes. Recent investigations have shown that impaired sympathetic innervation in the myocardium, as quantified by metaiodobenzylguanidine (MIBG) activity, is related to fatal clinical outcomes (3-7) and can predict the effects of drug treatment in patients with heart failure (8-13). Although cardiac autonomic function has been shown to be an independent powerful prognostic marker, arrhythmogenesis, lethal cardiac events, and long-term prognosis are multifactorial and have several known determinants: left ventricular ejection fraction (LVEF), a history of ischemia, the size of the ischemic burden, coronary risk factors, electrophysiologic indices, and the plasma concentration of brain natriuretic peptide (BNP). In other words, there is no single powerful predictor of arrhythmic death and effectiveness of prophylactic ICD use. Our previous studies showed that the prognostic value of cardiac MIBG activity can be improved in combination with other clinical variables, including LVEF and plasma BNP 
concentration, in patients with heart failure or left ventricular dysfunction (4-7).

However, it has not been determined whether cardiac autonomic function is useful for the selection of patients who are at greater risk for sudden death and who could benefit most from ICD use. The aims of this study were to determine, first, whether alterations of cardiac autonomic innervation quantified by cardiac MIBG imaging are related to lethal cardiac events, defined as an appropriate ICD shock against potentially fatal ventricular arrhythmias, and, second, whether assessment of cardiac sympathetic innervation and function in combination with conventional variables is useful for selection of patients who are at a higher risk for cardiac death and have the greatest need for ICD implantation.

\section{MATERIALS AND METHODS}

\section{Patient Population}

The study population consisted of 54 consecutive patients (mean age, 53 y; 40 men and 14 women) who met the American Heart Association/American College of Cardiology guidelines for ICD implantation (14) (Table 1). ICD implantation (Micro Jewel II, GEM, and GEM II; Medtronics Inc. or VENTAK MINI IV; Guidant Inc.) was done using a standard (subclavian) venous approach. Informed consent for ICD implantation and participation with prospective follow-up in this study was obtained on the basis of the guidelines of the ethics committee of our hospital. Clinical ventricular arrhythmias were confirmed by Holter monitors or by a standard electrocardiogram before admission of the patient at our hospital. In addition, all patients underwent Holter recordings during admission before ICD implantation. In 35 of the 54 patients, the dual-chamber pacing mode was used because of the presence of a combined atrioventricular block or atrial fibrillation. Table 1 shows the underlying cardiac diseases that were responsible for the lethal ventricular arrhythmias: 9 patients had idiopathic dilated cardiomyopathy, 6 had idiopathic ventricular fibrillation, 16 had secondary cardiomyopathy, 6 had arrhythmogenic right ventricular cardiomyopathy, 7 had Brugada syndrome, 2 had long-QT syndrome, and 8 had hypertrophic cardiomyopathy. Thus, primary or secondary cardiomyopathies were found in 39 patients; no organic heart disease was found in patients with Brugada syndrome, idiopathic ventricular fibrillation, or long-QT syndrome. The LVEF was assessed by 2-dimensional echocardiography within 2 wk of cardiac MIBG imaging. The mean LVEF was $48 \% \pm 17 \%$.

\section{Electrocardiographic Examinations}

The following electrophysiologic tests were performed before ICD implantation. Signal-averaged electrocardiography and an electrophysiologic study were performed on 40 and 41 of the 54 patients, respectively, to establish the diagnosis of lethal ventricular tachyarrhythmias and to determine whether ICD implantation was indicated. A ventricular tachyarrhythmia induction test was done in the standard manner and included burst pacing and double or triple paired pacing with a minimal coupling interval of $180 \mathrm{~ms}$ from both apical and outflow sites of the right ventricle. Ventricular tachycardia (VT) was defined as 3 or more consecutive ventricular extrasystoles; sustained VTwas defined as VT lasting for at least $30 \mathrm{~s}$. A positive late potential
TABLE 1

Patient Backgrounds and Underlying Diagnoses

\begin{tabular}{|c|c|}
\hline Parameter & Value \\
\hline Total patients $(n)$ & 54 \\
\hline Age (y) & $53 \pm 13$ \\
\hline \multicolumn{2}{|l|}{ Sex of the patient } \\
\hline Male $(n)$ & 40 \\
\hline Female $(n)$ & 14 \\
\hline LVEF (\%) & $48 \pm 17$ \\
\hline \multicolumn{2}{|l|}{ Underlying diagnosis } \\
\hline \multicolumn{2}{|l|}{ Organic heart disease } \\
\hline $\begin{array}{l}\text { Idiopathic dilated } \\
\text { cardiomyopathy }(n)\end{array}$ & 9 \\
\hline Hypertrophic cardiomyopathy* $(n)$ & 8 \\
\hline Secondary cardiomyopathy ${ }^{\dagger}(n)$ & 16 \\
\hline $\begin{array}{l}\text { Arrhythmogenic right ventricular } \\
\text { cardiomyopathy }(n)\end{array}$ & 6 \\
\hline \multicolumn{2}{|l|}{ Nonorganic heart disease } \\
\hline Brugada syndrome $(n)$ & 7 \\
\hline Long-QT syndrome $(n)$ & 2 \\
\hline Idiopathic ventricular fibrillation (n) & 6 \\
\hline Appropriate ICD discharge $(n)$ & 21 \\
\hline Sudden cardiac death $(n)$ & 3 \\
\hline $\begin{array}{l}\text { Acute myocardial infarction/other } \\
\text { cardiac death }(n)\end{array}$ & 0 \\
\hline Follow-up period (mo) & $15 \pm 11$ \\
\hline
\end{tabular}

${ }^{*}$ Four of 8 patients with hypertrophic cardiomyopathy were at dilated stage.

${ }^{\dagger}$ Coronary artery disease, 8 patients; cardiac sarcoidosis, 3 patients; polymyositis, 3 patients; Emery-Dreifuss muscular dystrophy, 1 patient; valvular heart disease, 1 patient.

Data are mean \pm SD.

on signal-averaged electrocardiography was defined as the presence of at least 2 of the following 3 criteria: a filtered QRS interval of more than $114 \mathrm{~ms}$, a root mean square of less than $20 \mu \mathrm{V}$, and a lowamplitude signal of more than $38 \mathrm{~ms}$.

\section{Cardiac MIBG Imaging}

Within 2 wk of ICD implantation but at least $3 \mathrm{~d}$ after electrocardiographic examinations, cardiac MIBG imaging was done using $111 \mathrm{MBq}$ of ${ }^{123} \mathrm{I}-\mathrm{MIBG}$ with a high specific activity (Daiichi Radioisotope Laboratories). Cardiac planar and tomographic MIBG images were obtained with the patient fasting and at rest $30 \mathrm{~min}$ (early) and $4 \mathrm{~h}$ (late) after intravenous injection of ${ }^{123}$ I-MIBG using a $\gamma$-camera equipped with a low-energy generalpurpose collimator. The view was anterior. Cardiac ${ }^{123}$ I-MIBG activity was quantified as heart-to-mediastinum (HMR) ratio by manually setting the region of interest on the upper mediastinum and the whole cardiac region using visual guidance-a technique that had previously been reported to have a high reproducibility (4-7). ${ }^{123}$ I-MIBG washout kinetics from the heart were also calculated as washout rate using a polar map technique with tomographic data because of the elimination of background activity, as previously reported (4-7).

\section{Measurements of Plasma Concentration of BNP}

Plasma concentration of BNP and atrial natriuretic peptide (ANP) were measured on the same day as or a few days after 
cardiac MIBG imaging. Samples used for the measurements were transferred to chilled disposable tubes containing aprotinin (500 kallikrein inactivator units $/ \mathrm{mL}$ ) and immediately placed on ice and centrifuged at $4{ }^{\circ} \mathrm{C}$. The concentration was measured by a specific immunoradiometric assay using a commercial kit (Shionogi), as previously reported (7); the kit contained 2 antihuman BNP monoclonal antibodies.

\section{Follow-up Protocol}

After discharge, all patients were prospectively followed at least every 3 mo for a mean of 15 mo at the outpatient pacemaker clinic of our university hospital, with the primary endpoint being lethal events consisting of cardiac death or appropriate ICD shock triggered by lethal arrhythmias (Table 1). According to documentation on appropriate ICD shock during follow-up, the patients were classified into 2 groups: a group with appropriate ICD shock or sudden cardiac death (group A) and a group with neither (group B). Standard drug therapy for arrhythmias, background diseases, or heart failure that had been used at study entry was continued as long as there were no adverse effects (Table 2). Antiarrhythmic agents, such as amiodarone, sotalol, class I antiarrhythmic drugs, or $\beta$-blockers, were used. Diuretics, digoxin, angiotensin-converting enzyme inhibitors, angiotensin receptor blockers, or $\beta$-blockers were used for heart failure, when indicated. An appropriate ICD discharge was defined as an electrical event occurring in response to sustained lethal VT or fibrillation, including defibrillation, cardioversion, and antitachycardia pacing, and was identified by electrocardiograms stored in the built-in memory system of the ICD.

\section{Statistical Analysis}

Statistical values are shown as mean \pm SD. Mean values were compared between the 2 groups using the unpaired $t$ test, and prevalence was compared using the $\chi^{2}$ test. A $P$ value of less than 0.05 was considered significant. Receiver operating characteristics were analyzed to determine optimal cutoffs for key parameters (i.e., late HMR, LVEF, and BNP) for predicting lethal arrhythmic events (Fig. 1). Hazard ratios and 95\% confidence intervals for the univariate model were computed using major clinical parameters. Multivariate analysis with a Cox proportional hazards model was also done using significant parameters identified by univariate analysis. These analyses were performed using a computer software program, the SPSS statistical program package (SPSS, version 11.0; SPSS Inc.).

TABLE 2

Comparison of Parameters Between Groups A and B

\begin{tabular}{|c|c|c|c|}
\hline Parameter & Group A $(n=21)$ & Group B $(n=33)$ & $P$ \\
\hline Age (y) & $53.4 \pm 16.2$ & $52.3 \pm 11.2$ & 0.7569 \\
\hline Sex of the patient & & & 0.9718 \\
\hline Male $(n)$ & 15 & 25 & \\
\hline Female $(n)$ & 6 & 8 & \\
\hline Organic heart disease (\%) & $16(76 \%)$ & $23(70 \%)$ & 0.8354 \\
\hline LVEF (\%) & $44 \pm 19$ & $51 \pm 15$ & 0.1284 \\
\hline LVDd (mm) & $56 \pm 12$ & $52 \pm 10$ & 0.1681 \\
\hline Early HMR of MIBG activity & $1.98 \pm 0.31$ & $2.13 \pm 0.31$ & 0.1047 \\
\hline Late HMR of MIBG activity & $1.75 \pm 0.44$ & $2.02 \pm 0.40$ & 0.0221 \\
\hline Washout rate $(\%)$ & $36 \pm 13$ & $28 \pm 14$ & 0.2821 \\
\hline Follow-up (mo) & $10 \pm 11$ & $18 \pm 11$ & 0.0279 \\
\hline SAECG filtered QRS & $133.0 \pm 31.7(n=15)$ & $121.7 \pm 17.0(n=25)$ & 0.1756 \\
\hline Root mean square & $22.8 \pm 17.7(n=15)$ & $34.2 \pm 78.4(n=25)$ & 0.6254 \\
\hline Low-amplitude signal & $38.6 \pm 15.6(n=15)$ & $39.6 \pm 13.9(n=25)$ & 0.8500 \\
\hline ANP & $66 \pm 54$ & $46 \pm 28$ & 0.0957 \\
\hline BNP & $199 \pm 188$ & $80 \pm 85$ & 0.0030 \\
\hline \multicolumn{4}{|l|}{ Concomitant medication } \\
\hline$\beta$-blocker & $67 \%(14 / 21)$ & $48 \%(16 / 33)$ & 0.3030 \\
\hline I-antiarrhythmic drug & $48 \%(10 / 21)$ & $24 \%(8 / 33)$ & 0.1388 \\
\hline Amiodarone & $43 \%(9 / 21)$ & $33 \%(11 / 33)$ & 0.6763 \\
\hline ACEI/ARB & $62 \%(13 / 21)$ & $36 \%(12 / 33)$ & 0.1199 \\
\hline Diuretic & $43 \%(9 / 21)$ & $24 \%(8 / 33)$ & 0.2563 \\
\hline Congestive heart failure & $57 \%(12 / 21)$ & $39 \%(13 / 33)$ & 0.3196 \\
\hline Positive SAECG $(n=40)$ & $67 \%(10 / 15)$ & $56 \%(14 / 25)$ & 0.7399 \\
\hline \multicolumn{4}{|l|}{ Electrophysiologic study $(n=41)$} \\
\hline \multicolumn{4}{|l|}{ Induction of VT/fibrillation } \\
\hline Sustained or nonsustained & $93 \%(14 / 15)$ & $92 \%(24 / 26)$ & 1.0000 \\
\hline Sustained & $80 \%(12 / 15)$ & $58 \%(15 / 26)$ & 0.1860 \\
\hline \multicolumn{4}{|l|}{ Clinical VT } \\
\hline Sustained or nonsustained & $100 \%(21 / 21)$ & $76 \%(25 / 33)$ & 0.0172 \\
\hline Sustained & $81 \%(17 / 21)$ & $61 \%(20 / 33)$ & 0.1429 \\
\hline
\end{tabular}

LVDd = left ventricular diameter; SAECG = signal-averaged electrocardiographic findings; ACEI = angiotensin-converting enzyme inhibitor; ARB = angiotensin receptor blocker. 


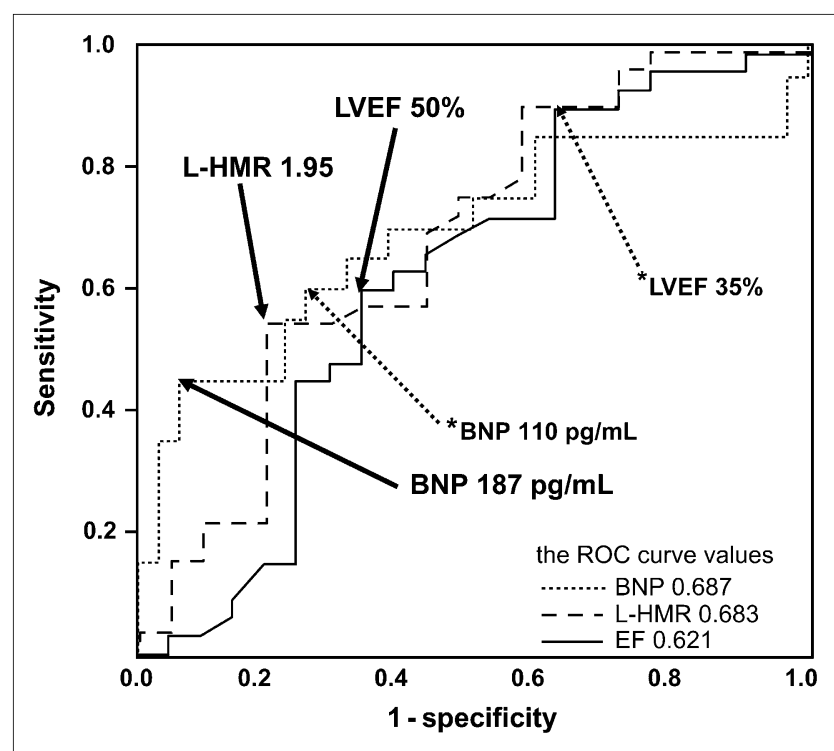

FIGURE 1. Receiver operating characteristic curves of cardiac late MIBG activity (HMR), LVEF, and BNP, indicating that optimal cutoffs for identifying lethal arrhythmic events are 1.95 for HMR, $50 \%$ for LVEF, and $187 \mathrm{pg} / \mathrm{mL}$ for BNP. Dotted arrows show suboptimal values (*) of LVEF (35\%) and BNP (110 pg/mL) that were used for subgroup analysis, for which results are shown in Figure 4. EF = ejection fraction.

\section{RESULTS}

In the 21 patients in group A, 3 sudden cardiac deaths were documented. Table 2 shows a comparison of the clinical backgrounds and electrophysiologic and imaging data of the patients in groups A and B before ICD implantation and at the start of follow-up. Compared with group B patients, group A patients had a significantly higher BNP level (199 \pm $188 \mathrm{pg} / \mathrm{mL}$ in group A vs. $80 \pm 85 \mathrm{pg} / \mathrm{mL}$ in group $\mathrm{B}, P=$ $0.0030)$, a significantly greater prevalence of VT $(100 \%$ in group A vs. $76 \%$ in group $\mathrm{B}, P=0.0172)$, and a significantly lower late HMR $(1.75 \pm 0.44$ in group A vs. $2.02 \pm 0.40$ in group $\mathrm{B}, P=0.0221$ ) (Table 2). Although the overall prevalence of VT in group A was significantly greater than that in group B (100\% vs. $76 \%, P=0.0172$, Table 2$)$, there was no significant difference in the prevalence of clinical sustained VT (Tables 2 and 3). No significant difference was found in other variables, including age, LVEF, left ventricular diameter, medications, and electrophysiologic data. When all concomitant medications were lumped together, however, group A patients were found to have undergone drug treatment more frequently than had group B patients: 20 (95\%) vs. $21(64 \%), P=0.0092$. Table 4 summarizes the results of univariate logistic regression analysis for all patients. Plasma BNP level, medication (which was defined as pharmacologic treatment with any drug), and the late HMR of cardiac MIBG activity were identified as significant predictors. In addition, multivariate analysis with a Cox proportional hazards model revealed late HMR to be an independent predictor of lethal arrhythmic events (Table 5).
Figure 2 shows 2 typical planar images from groups A and B. The patient in group A had markedly decreased LVEF, markedly decreased cardiac MIBG activity (1.16), and a mildly elevated BNP level, whereas the patient in group B had nearly normal values for LVEF, cardiac MIBG activity (HMR, 2.30), and BNP. When the data of only the 39 patients with organic heart disease were analyzed (Table 3), compared with group B patients, group A patients had a significantly higher BNP level $(243 \pm 186 \mathrm{pg} / \mathrm{mL}$ in group A vs. $101 \pm 92 \mathrm{pg} / \mathrm{mL}$ in group $\mathrm{B}, P=0.0032$ ), a significantly lower late HMR $(1.60 \pm 0.32$ in group A vs. $1.87 \pm 0.35$ in group B, $P=0.0184$ ), and a significantly higher MIBG washout rate $(40 \% \pm 11 \%$ in group A vs. $31 \% \pm 14 \%$ in group B, $P=$ 0.0409). There were no significant differences in other variables, though group A patients tended to have an ANP level that was higher and an LVEF that was lower than those of group B patients.

Three key parameters-late HMR, LVEF, and plasma BNP level-were selected on the basis of the results of logistic regression analysis (Table 4), the results of our previous studies (4-7), and the American Heart Association/American College of Cardiology guidelines for ICD implantation (14). The combined diagnostic values were analyzed for identifying patients with lethal cardiac events (sudden cardiac death and ICD shock). The variable thresholds were determined by ROC analysis (Fig. 1) using the 3 key parameters (1.95 for HMR, $50 \%$ for LVEF, and $187 \mathrm{pg} / \mathrm{mL}$ for BNP), and scatterplots of 2 combined parameters were generated (Fig. 3). Patients with an LVEF of less than $50 \%$ and a late HMR of less than 1.95 had ICD discharge or sudden cardiac death more frequently than did other patients: 14 of $24(58.3 \%)$ vs. 7 of $30(23.3 \%), P=$ 0.0192 (Fig. 3A). When only patients with organic heart disease were considered, patients with an LVEF of less than $50 \%$ and a late HMR of less than 1.95 had ICD discharge or sudden cardiac death more frequently than did other patients: 14 of $24(58.3 \%)$ vs. 2 of 15 (13.3\%), $P=0.0077$ (Fig. 3B). Similarly, in patients with a BNP level of greater than $187 \mathrm{pg} /$ $\mathrm{mL}$ and a late HMR of less than 1.95, ICD discharge or sudden cardiac death was observed more frequently than in other patients (Fig. 3C): 9 of 11 (81.8\%) vs. 11 of 41 (26.8\%), $P=$ 0.0015 . When only patients with organic heart disease were considered, patients with a late HMR of less than 1.95 and a BNP of greater than $187 \mathrm{pg} / \mathrm{mL}$ had more frequent ICD discharge than did other patients: 9 of $11(81.8 \%)$ vs. 7 of 28 (25\%), $P=0.0026$ (Fig. 3D). The overall predictive values of combinations were as follows: positive predictive values, $82 \%$ for HMR + BNP and 58\% for HMR + LVEF; negative predictive values, $73 \%$ for HMR + BNP and $77 \%$ for HMR + LVEF; sensitivities, $45 \%$ for HMR + BNP and $67 \%$ for HMR + LVEF; and specificities, $94 \%$ for HMR + BNP and $70 \%$ for HMR + LVEF.

Figure 4 shows the lethal event rates correlated with the combined use of late HMR and LVEF or BNP in the patient subgroups. Overall, the event rate increased as late HMR decreased. However, the highest event rates $(83 \%-100 \%)$ occurred when the late HMR was 1.39 or less, independent of 
TABLE 3

Comparison of Parameters in 39 Patients with Organic Heart Diseases from Groups A and B

\begin{tabular}{|c|c|c|c|}
\hline Parameter & Group A $(n=16)$ & Group B $(n=23)$ & $P$ \\
\hline Age $(y)$ & $57.6 \pm 12.7$ & $52.4 \pm 10.9$ & 0.1790 \\
\hline Sex of the patient & & & 1.0000 \\
\hline Male $(n)$ & 12 & 18 & \\
\hline Female $(n)$ & 4 & 5 & \\
\hline LVEF (\%) & $37 \pm 16$ & $46 \pm 14$ & 0.0857 \\
\hline LVDd (mm) & $60 \pm 11$ & $55 \pm 10$ & 0.1288 \\
\hline Early HMR of MIBG activity & $1.93 \pm 0.33$ & $2.07 \pm 0.33$ & 0.2060 \\
\hline Late HMR of MIBG activity & $1.60 \pm 0.32$ & $1.87 \pm 0.35$ & 0.0184 \\
\hline Washout rate (\%) & $40 \pm 11$ & $31 \pm 14$ & 0.0409 \\
\hline Follow-up (mo) & $10 \pm 11$ & $18 \pm 11$ & 0.0475 \\
\hline SAECG filtered QRS & $143.4 \pm 34.3$ & $126.6 \pm 17.3$ & 0.1220 \\
\hline Root mean square & $23.8 \pm 18.0$ & $40.7 \pm 93.8$ & 0.6225 \\
\hline Low-amplitude signal & $39.9 \pm 17.8$ & $39.3 \pm 15.0$ & 0.9323 \\
\hline ANP & $73 \pm 56$ & $47 \pm 30$ & 0.0676 \\
\hline BNP & $243 \pm 186$ & $101 \pm 92$ & 0.0032 \\
\hline \multicolumn{4}{|l|}{ Concomitant medication } \\
\hline$\beta$-blocker & $68.8 \%(11 / 16)$ & $60.9 \%(14 / 23)$ & 0.8873 \\
\hline I-antiarrhythmic drug & $43.8 \%(7 / 16)$ & $34.8 \%(8 / 23)$ & 0.8168 \\
\hline Amiodarone & $56.3 \%(9 / 16)$ & $47.8 \%(11 / 23)$ & 0.8477 \\
\hline ACEI/ARB & $75.0 \%(12 / 16)$ & $52.2 \%(12 / 23)$ & 0.1923 \\
\hline Diuretic & $56.3 \%(9 / 16)$ & $34.8 \%(8 / 23)$ & 0.3165 \\
\hline Congestive heart failure & $75.0 \%(12 / 16)$ & $56.5 \%(13 / 23)$ & 0.3171 \\
\hline Positive SAECG $(n=28)$ & $70.0 \%(7 / 10)$ & $61.1 \%(11 / 18)$ & 0.7029 \\
\hline \multicolumn{4}{|l|}{ Electrophysiologic study $(n=29)$} \\
\hline \multicolumn{4}{|l|}{ Induction of VT/fibrillation } \\
\hline Sustained or nonsustained & $100 \%(12 / 12)$ & $100 \%(17 / 17)$ & 1.0000 \\
\hline Sustained & $83.3 \%(10 / 12)$ & $52.9 \%(9 / 17)$ & 0.1261 \\
\hline \multicolumn{4}{|l|}{ Clinical VT } \\
\hline Sustained or nonsustained & $100 \%(16 / 16)$ & $91.3 \%(21 / 23)$ & 0.5034 \\
\hline Sustained & $87.5 \%(14 / 16)$ & $78.3 \%(18 / 23)$ & 0.6776 \\
\hline
\end{tabular}

LVDd = left ventricular diameter; SAECG = signal-averaged electrocardiographic findings; ACEI = angiotensin-converting enzyme inhibitor; ARB = angiotensin receptor blocker.

LVEF, whereas the event rates were the lowest (0\%-20\%) among subgroups with a late HMR of at least 1.95 (Fig. 4A). The event rates were intermediate (about 40\%-50\%) when the late HMR was between 1.40 and 1.94 but increased to $80 \%$ when the LVEF was less than $35 \%$ together with a late HMR of between 1.40 and 1.74. Similarly, independent of plasma BNP concentration, event rates were relatively high $(75 \%-$ $83 \%$ ) when the late HMR was 1.39 or less (Fig. 4B). Event rates were intermediate (about 43\%-50\%) when the late HMR was 1.40 or more but were higher $(75 \%-100 \%)$ when the plasma BNP level was more than $187 \mathrm{pg} / \mathrm{mL}$ in patients with a late HMR of between 1.40 and 1.94 .

\section{DISCUSSION}

By correlating documented ICD shock during follow-up with cardiac function indices, this study showed that impairment of cardiac sympathetic innervation could predict lethal cardiac events, contributing to the identification of patients who would receive substantial and minimal benefits from prophylactic ICD implantation.

\section{Sudden Cardiac Death and Cardiac Sympathetic Nerve Function}

Several studies have shown that altered autonomic function is responsible for ventricular arrhythmias or sudden cardiac death. Earlier studies using cardiac neurotransmitter imaging (15-17) also demonstrated that impaired uptake of norepinephrine analogs was related to severe ventricular tachyarrhythmias. However, it was still necessary to determine whether altered cardiac MIBG activity is associated with fatal cardiac episodes due to ventricular tachyarrhythmias. At an advanced stage of heart failure, the plasma norepinephrine level and the firing of autonomic nerves in skeletal muscles are markedly increased, whereas heart rate variability and cardiac sympathetic innervation and function are greatly depressed (18-20). These complex changes in autonomic nervous function show that sympathetic innervation acts differently at different functional sites under various conditions, indicating the need for direct assessment of cardiac autonomic innervation. In the present study, the occurrence of documented ICD shock due to fatal arrhythmias during follow-up correlated with cardiac MIBG activity, left 
TABLE 4

Univariate Logistic Regression Analysis in All Patients

\begin{tabular}{|c|c|c|c|c|c|}
\hline \multirow[b]{2}{*}{ Variable } & \multirow[b]{2}{*}{ Wald $\chi 2$} & \multirow[b]{2}{*}{ Hazard ratio } & \multicolumn{2}{|c|}{$95 \% \mathrm{Cl}$ of hazard ratio } & \multirow[b]{2}{*}{$P$} \\
\hline & & & Lower & Upper & \\
\hline BNP & 6.378 & 1.007 & 1.002 & 1.012 & 0.012 \\
\hline Medication & 5.025 & 11.429 & 1.358 & 96.163 & 0.025 \\
\hline Late HMR & 4.761 & 0.182 & 0.040 & 0.841 & 0.029 \\
\hline Washout rate & 3.780 & 1.051 & 1.000 & 1.105 & 0.052 \\
\hline Previous myocardial infarction & & & & & 0.061 \\
\hline Early HMR & & & & & 0.109 \\
\hline ANP & & & & & 0.116 \\
\hline LVEF & & & & & 0.129 \\
\hline LVDd & & & & & 0.169 \\
\hline \multicolumn{6}{|l|}{ Clinical VT } \\
\hline Sustained or nonsustained & & & & & 0.999 \\
\hline Sustained & & & & & 0.117 \\
\hline \multicolumn{6}{|l|}{ Induction of VT/fibrillation } \\
\hline Sustained or nonsustained & & & & & 0.903 \\
\hline Sustained & & & & & 0.147 \\
\hline Amiodarone & & & & & 0.556 \\
\hline SAECG filtered QRS & & & & & 0.188 \\
\hline Root mean square & & & & & 0.637 \\
\hline Low-amplitude signal & & & & & 0.845 \\
\hline
\end{tabular}

ventricular function, and plasma BNP level. These 3 variables were found to contribute to identification of patients at high risk for sudden cardiac death (Tables 2 and 3). Electrophysiologic indices, including the inducibility of lethal ventricular tachyarrhythmias and signal-averaged electrocardiography parameters, were not useful in helping to select patients who had the greatest risk for sudden cardiac death. Arora et al. (20) showed prospectively that appropriate ICD shock most likely occurs when both cardiac MIBG activity and heart rate variability are highly depressed. Microvolt T-wave alternans has recently been noted not only for its usefulness in arrhythmia risk assessment but also for its potential usefulness in the identification of patients who can benefit most from prophylactic ICD therapy $(21-26)$. This method is simple, practical, and probably cost-effective (22). An increase in repolarization instability assessed by

TABLE 5

Multivariate Analysis Using Cox Hazard Proportional Model in All Patients

\begin{tabular}{lccccc}
\hline & & & \multicolumn{3}{c}{$\begin{array}{c}95 \% \mathrm{Cl} \text { of } \\
\text { hazard ratio }\end{array}$} \\
\cline { 3 - 5 } Variable & Wald $\chi 2$ & Hazard ratio & Lower & Upper & $P$ \\
\hline Late HMR & 6.989 & 0.141 & 0.033 & 0.603 & 0.008 \\
BNP & & & & 0.194 \\
Medication & & & 0.140 \\
\hline & & & & \\
\hline $\mathrm{Cl}=$ confidence interval. & & & \\
\hline
\end{tabular}

T-wave alternans precedes ventricular tachyarrhythmias culminating in sudden cardiac death (23). T-wave alternans, however, is one of the indices of repolarization instability and has a complex spectrum that varies dynamically over short intervals. In addition to differences in measurements and definitions of T-wave alternans (26), averaged measurements are possibly affected by various factors, including artifacts caused by baseline wander due to perspiration, motion, and respiratory oscillation; heart rate or rhythm disturbance; systemic or central (noncardiac) autonomic
A

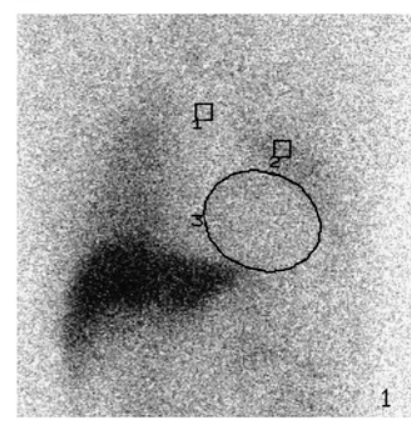

B

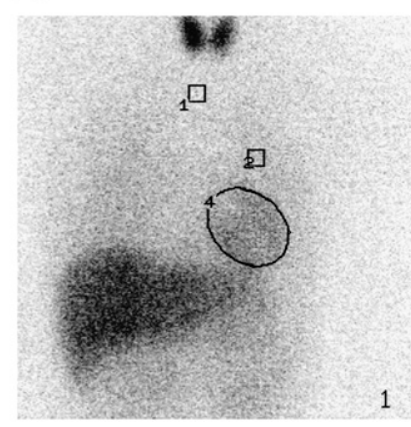

FIGURE 2. Typical cardiac MIBG images from groups $A$ and B. (A) A 69-y-old man with previous myocardial infarction who had slightly increased level of BNP (80 pg/mL), markedly reduced LVEF (19\%), and markedly reduced late HMR of MIBG activity (1.16). (B) A 20-y-old man with Brugada syndrome in whom LVEF (67\%), plasma BNP level (34 pg/mL), and late HMR (2.30) were nearly normal. $1=$ mediastinal region of interest; 2 $=$ lung region of interest; 3 and $4=$ heart region of interest. 


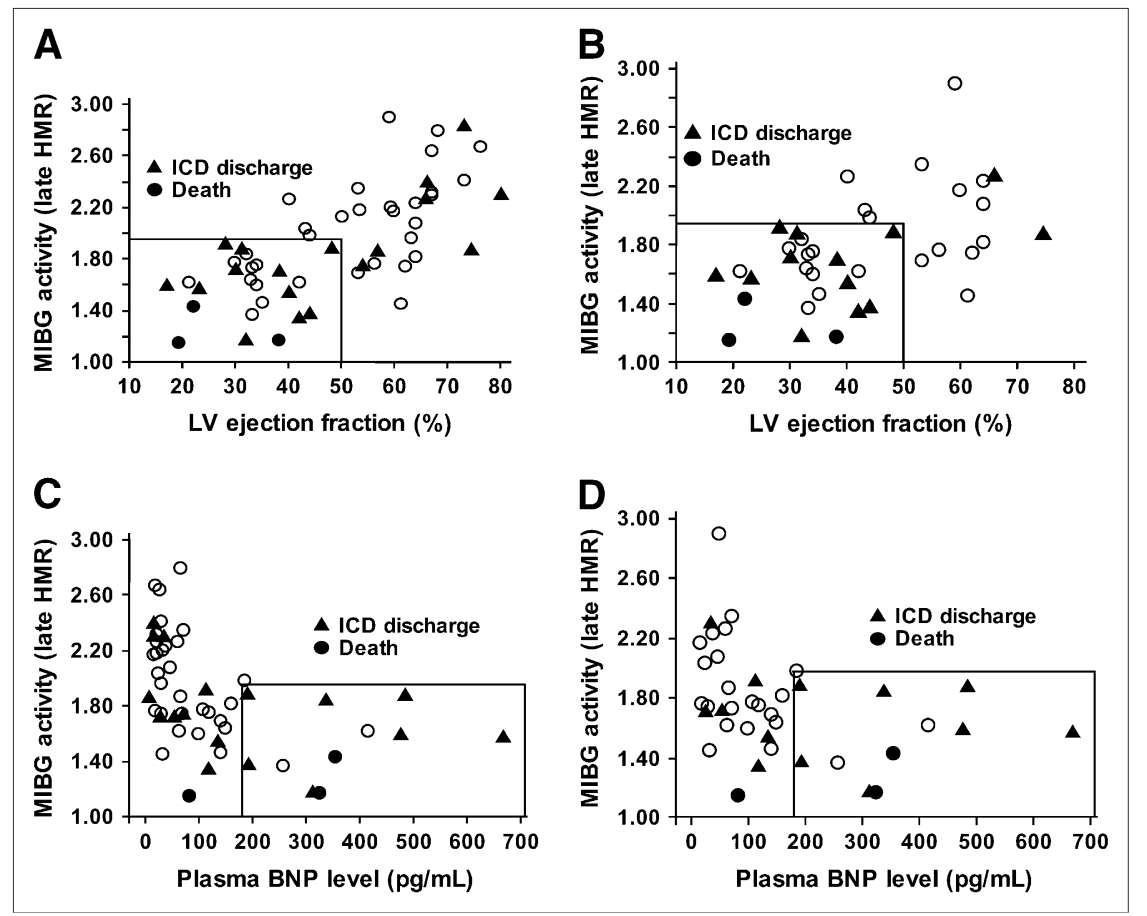

FIGURE 3. Scatterplots of cardiac MIBG activity (late HMR) in combination with LVEF and plasma BNP concentration. (A) In all 54 patients, patients with late HMR of less than 1.95 and LVEF of less than $50 \%$ had significantly $(P=0.0192)$ greater prevalence of lethal events (58.3\%) than did other patients (23.3\%). Sudden cardiac death occurred in 3 patients, 18 patients had appropriate ICD shock, and 33 patients had neither ICD shock nor cardiac death (O). (B) In 39 patients with organic heart disease, patients with late HMR of less than 1.95 and LVEF of less than $50 \%$ had significantly $(P=0.0077)$ greater prevalence of lethal events $(58.3 \%)$ than did other patients (13.3\%). (C) In all 52 patients, patients with late HMR of less than 1.95 and BNP of greater than $187 \mathrm{pg} / \mathrm{mL}$ had significantly greater prevalence of lethal events than did other patients: $81.8 \%$ vs. $26.8 \%, P=$ 0.0015 . (D) In 39 patients with organic heart disease, patients with late HMR of less than 1.95 and BNP of greater than 187 $\mathrm{pg} / \mathrm{mL}$ had significantly greater prevalence of lethal events than did other patients: $81.8 \%$ vs. $25 \%, P=0.0026$. tone; circulating catecholamines; drugs used; and underlying cardiac diseases $(23,24)$. It remains to be established whether, like an upsurge in T-wave alternans or other forms of repolarization instabilities preceding spontaneous arrhythmic events in humans $(23,25)$, an averaged T-wave alternans frequency assessed by ambulatory recording is directly related to and can predict arrhythmic death in a prospective cohort study. On the other hand, cardiac MIBG activity is highly specific for sympathetic innervation in the myocardium per se and is reproducible because of the specific active transport, storage, and release systems at nerve endings. Because microvolt $\mathrm{T}$-wave alternans testing, however, has high negative predictive powers, there is a need to investigate whether the presented scintigraphic technique has an incremental prognostic value in combination with T-wave alternans testing.

The mechanisms underlying impaired cardiac presynaptic sympathetic innervation that are responsible for lethal arrhythmias remain to be investigated. The changes in preand postsynaptic autonomic function in the failing heart are probably dependent on the progression of heart failure and structural myocardial damage (27). Cardiac MIBG activity assessed several hours after tracer injection has been shown to be related to myocardial norepinephrine content, left ventricular systolic function, and New York Heart Association functional status. Both ischemic and nonischemic etiologies can be responsible for the impairment of cardiac MIBG activity (5). In patients with organic heart disease, the ICD shock group had a significant increase in cardiac MIBG washout kinetics, compared with those who had no ICD shock. This finding is consistent with accelerated norepinephrine release and decreased uptake at presynaptic nerve endings, leading to spillover of norepinephrine (MIBG) in patients with moderate heart failure (28). In this group, an increase in cardiac sympathetic dynamics may facilitate arrhythmogenicity by mechanisms involving a reentry circuit, automaticity, or triggered activity. The heterogeneous degeneration process in viable but failing myocardial tissue is also related to exacerbation of electrical instability and causes denervation supersensitivity to sympathetic stimulation that precipitates ventricular arrhythmias $(29,30)$. Thus, both functional and structural impairments of cardiac sympathetic innervation assessed by MIBG activity are likely to be related to lethal arrhythmic events.

\section{Further Risk Stratification of ICD Patients Using Cardiac Sympathetic Activity}

Cardiac MIBG activity was closely related both to a low event rate and to a high event rate depending on HMR, but it was not related to an intermediate event rate. These data suggest the limited prognostic efficacy of 1 variable and highlight the necessity of using combinations of other clinical parameters to risk-stratify patients at an intermediate risk for arrhythmia death. The data presented here showed that combined use of LVEF or BNP with cardiac MIBG activity could more effectively evaluate the need for ICD treatment in patients who have moderate impairment of cardiac sympathetic innervation. Triggers of lethal tachyarrhythmias or sudden cardiac death are multifactorial. Such triggers include the etiology of heart failure, cardiac systolic function, hemodynamics, plasma BNP level, concomitant drug use, and noncardiac complications. Patients at an intermediate 


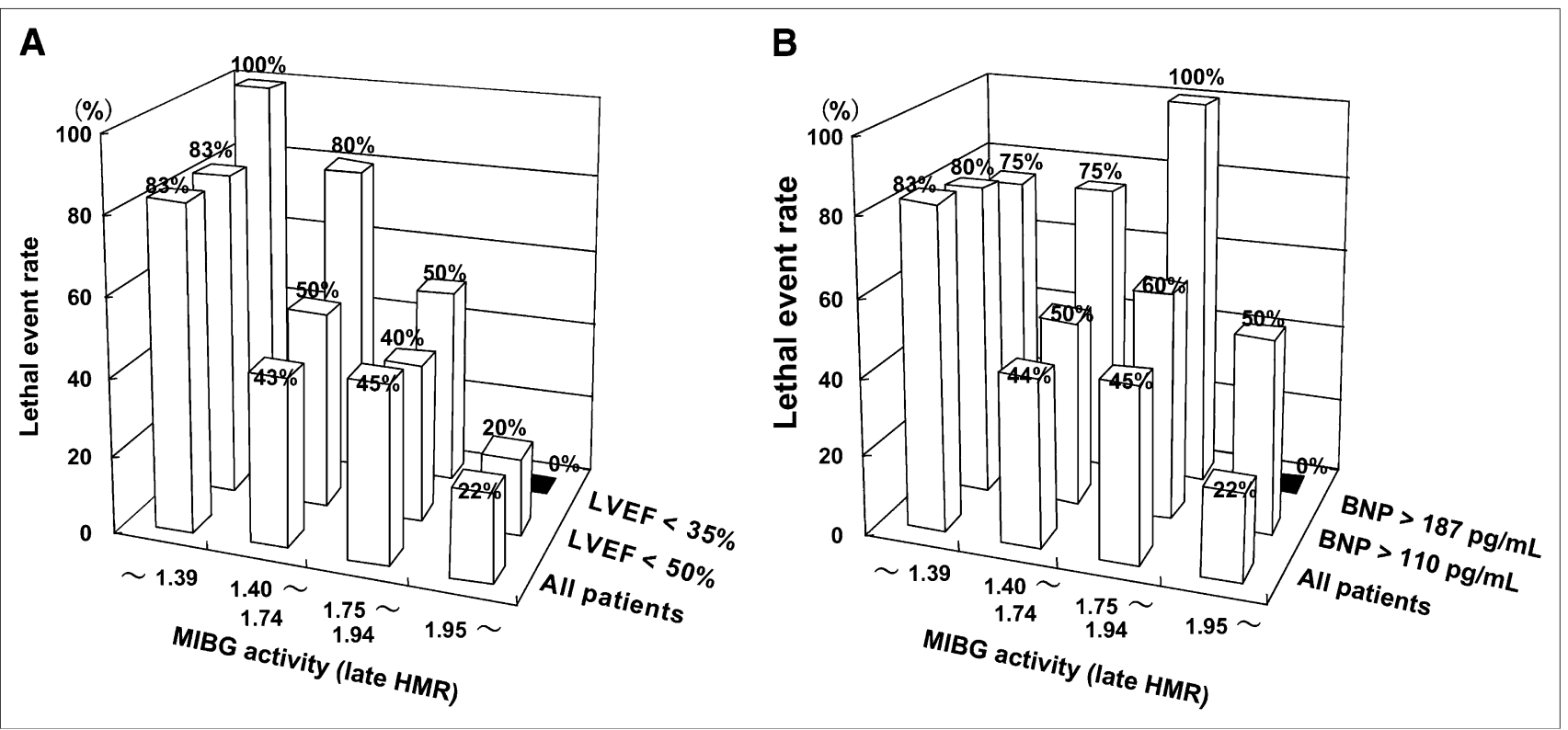

FIGURE 4. Lethal event rates among quartiles of late HMR when optimal and suboptimal cutoffs of LVEF and plasma BNP level were considered. (A) Independent of LVEF, event rates were highest (83\%-100\%) when late HMR was 1.39 or less. Conversely, event rates were lowest (0\%-22\%) among subgroups when late HMR was 1.95 or more. When late HMR was between 1.40 and 1.94, event rates were intermediate (about 40\%-50\%), but event rate was high (80\%) when LVEF was less than $35 \%$. (B) Independent of plasma BNP concentration, event rates were relatively high (75\%-83\%) when late HMR was 1.39 or less. Although event rates were intermediate (about 44\%-60\%) when late HMR was 1.40 or more, event rate was high (75\%-100\%) when plasma BNP level was more than $187 \mathrm{pg} / \mathrm{mL}$.

risk for lethal arrhythmic events are most likely affected by these background factors. Sheldon et al. (31) reported that the patients at highest risk of death who benefit most from ICD therapy could be identified on the basis of age, LVEF, and New York Heart Association status. Our previous studies (47) have also shown that reduced cardiac MIBG activity is an independent determinant of long-term prognosis and has better prognostic value when used in combination with age, LVEF, history of previous myocardial infarction, New York Heart Association status, presence of noncardiac complications, such as renal dysfunction or diabetes mellitus, and plasma BNP level. These findings support the rationale for the use of several different cardiac indices that are related to lethal cardiac events. Overall, LVEF was less associated with lethal arrhythmic events in combination with cardiac MIBG activity than was BNP level when combined with cardiac MIBG activity in this study. When cardiac MIBG was moderately reduced, however, LVEF effectively identified less than $35 \%$ of patients at an increased risk for events. The present and previous results indicate that a further large-scale investigation is needed to establish the most effective method for risk stratification using significant clinical parameters in combination with cardiac MIBG indices and to show the incremental predictive powers.

\section{Limitations}

The present results revealed not only the pathophysiologic implications of impaired cardiac sympathetic innervation in patients at risk for lethal cardiac events or sudden death but also the feasibility for further clarifying the risk of lethal outcomes in patients treated with ICD implantation. Besides BNP and late HMR, the washout rate of MIBG activity and a history of prior myocardial infarction were close to being statistically significant (Table 4). Our previous study (4) showed the prognostic values of prior myocardial infarction when combined with late HMR. Other investigators $(8,11,12)$ have demonstrated the possibility that washout rate is a prognostic marker. Given our encouraging results and previous findings, a large-scale, long-term study should be done to reach more definitive conclusions regarding the efficacies of the presented techniques. In particular, it is critical to find a method that has a maximal negative predictive value, because underuse, as well as overuse, of the ICD has to be avoided so as to minimize the possibility of fatal outcomes without ICD therapy and to maximize the costeffectiveness of this device. To accomplish this requirement, long-term follow-up and careful management of patients are necessary. In addition, the indications for ICD implantation need to be appropriately revised. There is also a need to increase the availability of cardiac ${ }^{123}$ I-MIBG imaging. The noninvasive features and simple imaging protocol, as well as the quantification of cardiac sympathetic innervation, of cardiac ${ }^{123}$ I-MIBG imaging could contribute to its widespread clinical application in patients with ICD implantation who have heart failure and are at a mild-to-moderate risk for lethal cardiac events. Finally, because there are several factors that may affect cardiac MIBG activity, such as electrophysiologic provocation testing with cardioversion, 
drug treatment (8-13), and progression of underlying cardiac disease, repeated imaging may be necessary for more precise estimation of the activity during a long-term follow-up.

\section{CONCLUSION}

Altered cardiac autonomic innervation, as assessed by cardiac MIBG activity, is closely related to lethal cardiac events, including ICD shock for malignant tachyarrhythmias and sudden cardiac death. In combination with plasma BNP concentration or cardiac function, the assessment of cardiac MIBG activity can help precisely identify patients who are at greater risk for fatal arrhythmias and who would benefit most from an ICD. Assessment of cardiac MIBG activity would also contribute to more appropriate use of ICD implantation and may help predict and prevent further lethal cardiac episodes.

\section{REFERENCES}

1. Connolly SJ, Hallstrom AP, Cappato R, et al. Meta-analysis of the implantable cardioverter defibrillator secondary prevention trials. AVID, CASH and CIDS Studies. Antiarrhythmics vs Implantable Defibrillator Study. Cardiac Arrest Study Hamburg. Canadian Implantable Defibrillator Study. Eur Heart J. 2000;21:2071-2078.

2. Moss AJ, Zareba W, Hall WJ, et al., for the Multicenter Automatic Defibrillator Implantation Trial II Investigators. Prophylactic implantation of a defibrillator in patients with myocardial infarction and reduced ejection fraction. $N$ Engl J Med. 2002;346:877-883.

3. Merlet P, Valette H, Dubois-Randé JL, et al. Prognostic value of cardiac MIBG imaging in patients with heart failure. J Nucl Med. 1992;33:471-477.

4. Nakata T, Miyamoto K, Doi A, et al. Cardiac death prediction and impaired cardiac sympathetic innervation assessed by MIBG in patients with failing and non-failing hearts. J Nucl Cardiol. 1998;5:579-590.

5. Wakabayashi T, Nakata T, Hashimoto A, et al. Assessment of underlying etiology and cardiac sympathetic innervation to identify patients at high risk of cardiac death. J Nucl Med. 2001;42:1757-1767.

6. Nakata T, Wakabayashi T, Kyuma M, et al. Prognostic implications of an initial loss of cardiac metaiodobenzylguanidine uptake and diabetes mellitus in patients with left ventricular dysfunction. J Card Fail. 2003;9:113-121.

7. Kyuma M, Nakata T, Hashimoto A, et al. Incremental prognostic implications of brain natriuretic peptide, cardiac sympathetic nerve innervation and non-cardiac disorders in patients with heart failure. $J$ Nucl Med. 2004;45:155-163.

8. Suwa M, Otake Y, Moriguchi A, et al. Iodine-123 metaiodobenzylguanidine myocardial scintigraphy for prediction of response to beta-blocker therapy in patients with dilated cardiomyopathy. Am Heart J. 1997;133:353-358.

9. Toyama T, Aihara Y, Iwasaki T, et al. Cardiac sympathetic activity estimated by ${ }^{123}$ I-MIBG myocardial imaging in patients with dilated cardiomyopathy after beta-blocker or angiotensin-converting enzyme inhibitor therapy. J Nucl Med. 1999;40:217-223.

10. Agostini D, Belin A, Amar MH, et al. Improvement of cardiac neuronal function after carvedilol treatment in dilated cardiomyopathy: a ${ }^{123} \mathrm{I}-\mathrm{MIBG}$ scintigraphic study. J Nucl Med. 2000;41:845-851.

11. Kasama S, Toyama T, Kumakura H, et al. Addition of valsartan to an angiotensinconverting enzyme inhibitor improves cardiac sympathetic nerve activity and left ventricular function in patients with congestive heart failure. J Nucl Med. 2003; 44:884-890.

12. Kasama S, Toyama T, Kumakura H, et al. Effect of spironolactone on cardiac sympathetic nerve activity and left ventricular remodeling in patients with dilated cardiomyopathy. J Am Coll Cardiol. 2003;41:574-581.
13. Nakata T, Wakabayashi T, Kyuma K, Takahashi T, Tsuchihashi K, Shimamoto K. Cardiac metaiodobenzylguanidine activity can predict the long-term prognostic efficacy of angiotensin-converting enzyme inhibitors and/or beta-adrenoceptor blockers in patients with heart failure. Eur J Nucl Med Mol Imaging. 2005;32: 186-194.

14. Gregoratos G, Cheitlin MD, Conill A, et al. ACC/AHA guidelines for implantation of cardiac pacemakers and antiarrhythmia devices: executive summary-a report of the ACC/AHA task force on practice guidelines (committee on pacemaker implantation). Circulation. 1998;97:1325-1335.

15. Schwartz PJ, La Rovere MT, Vanoli E. Autonomic nervous system and sudden cardiac death: experimental basis and clinical observations for post-myocardial infarction risk stratification. Circulation. 1992;85:I77-I91.

16. Gill JS, Hunter GJ, Gane J, Ward DE, Camm AJ. Asymmetry of cardiac $\left[{ }^{123} \mathrm{I}\right]$ metaiodobenzylguanidine scans in patients with ventricular tachycardia and a "clinically normal" heart. Br Heart J. 1993;69:6-13.

17. Calkins H, Allman K, Bolling S. Correlation between scintigraphic evidence of regional sympathetic neuronal dysfunction and ventricular refractoriness in the human heart. Circulation. 1993;88:172-179.

18. Galinier M, Pathak A, Fourcade J, et al. Depressed low frequency power of heart rate variability as an independent predictor of sudden death in chronic heart failure. Eur Heart J. 2000;21:475-482.

19. La Rovere MT, Pinna GD, Maestri R, et al. Short-term heart rate variability strongly predicts sudden cardiac death in chronic heart failure patients. Circulation. 2003;107:565-570.

20. Arora R, Ferrick KJ, Nakata T, et al. I-123 MIBG imaging and heart rate variability analysis to predict the need for implantable cardioverter defibrillator. J Nucl Cardiol. 2003;10:121-131.

21. Rosenbaum DS, Jackson LE, Smith JM, Garan H, Ruskin JN, Cohen RJ. Electrical alternans and vulnerability to ventricular arrhythmias. N Engl J Med. 1994; 330:235-241.

22. Chan PS, Stein K, Chow T, Fendrick M, Bigger JT, Vijan S. Cost-effectiveness of a microvolt T-wave alternans screening strategy for implantable cardioverterdefibrillator placement in the MADIT-II-eligible population. J Am Coll Cardiol. 2006;48:112-121.

23. Shusterman V, Goldberg A, London B. Upsurge in T-wave alternans and nonalternating repolarization instability precedes spontaneous initiation of ventricular tachyarrhythmias in humans. Circulation. 2006;113:2880-2887.

24. Kaufman ES, Mackall JA, Julka B, Drabek C, Rosenbaum DS. Influence of heart rate and sympathetic stimulation on arrhythmogenic T wave alternans. Am J Physiol Heart Circ Physiol. 2000;279:H1248-H1255.

25. Armoundas AA, Albert CM, Cohen RJ, Mela T. Utility of implantable/ cardioverter defibrillator electrocardiograms to estimate repolarization alternans preceding a tachyarrhythmic event. J Cardiovasc Electrophysiol. 2004;15:594597.

26. Verrier RL, Nearing BD, Kwaku KF. Noninvasive sudden death risk stratification by ambulatory ECG-based $\mathrm{T}$ wave alternans analysis: evidence and methodological guidelines. Ann Noninvasive Electrocardiol. 2005;10:110-120.

27. Nakata T, Wakabayashi T, Nagahara D. Assessment of cardiac innervation in heart failure and lethal arrhythmias: Therapeutic and prognostic implications. Curr Cardiol Rev. 2005;1:29-36.

28. Eisenhofer G, Friberg P, Rundqvist B, et al. Cardiac sympathetic nerve function in congestive heart failure. Circulation. 1996;93:1667-1676.

29. Miyazaki T, Zipes DP. Presynaptic modulation of efferent sympathetic and vagal neurotransmission in the canine heart by hypoxia, high $\mathrm{K}+$, low $\mathrm{pH}$, and adenosine: possible relevance to ischemia-induced denervation. Circ Res. 1990;66: 289-301.

30. Inoue H, Zipes DP. Result of sympathetic denervation in the canine heart: supersensitivity that may be arrhythmogenic. Circulation. 1987;75:877887.

31. Sheldon R, Connolly S, Krahn A, Roberts R, Gent M, Gardner M. Identification of patients most likely to benefit from implantable cardioverter-defibrillator therapy. The Canadian Implantable Defibrillator Study. Circulation. 2000;101: $1660-1664$. 